Chapter 3

\title{
Transhepatic Bile Acid Kinetics in Pigs and Humans
}

\author{
Hannah M. Eggink \\ F. Samuel van Nierop \\ Marieke G. Schooneman \\ Anita Boelen \\ Andries Kalsbeek \\ Martijn Koehorst \\ Gabriella A.M. Ten Have \\ L. Maurits de Brauw \\ Albert K. Groen \\ Johannes A. Romijn \\ Nicolaas E.P. Deutz \\ Maarten R. Soeters
}

Clinical Nutrition 2017 


\section{ABSTRACT}

Background \& aims: Bile acids (BAs) play a key role in lipid uptake and metabolic signalling in different organs including gut, liver, muscle and brown adipose tissue. Portal and peripheral plasma BA concentrations increase after a meal. However, the exact kinetics of postprandial BA metabolism have never been described in great detail. We used a conscious porcine model to investigate postprandial plasma concentrations and transorgan fluxes of BAs, glucose and insulin using the para-aminohippuric acid dilution method. Methods: Eleven pigs with intravascular catheters received a standard mixed-meal while blood was sampled from different veins such as the portal vein, abdominal aorta and hepatic vein. To translate the data to humans, fasted venous and portal blood was sampled from non-diabetic obese patients during gastric by-pass surgery. Results: The majority of the plasma bile acid pool and postprandial response consisted of glycine-conjugated forms of primary bile acids. Conjugated bile acids were more efficiently cleared by the liver than unconjugated forms. The timing and size of the postprandial response showed large interindividual variability for bile acids compared to glucose and insulin. Conclusions: The liver selectively extracts most BAs and BAs with highest affinity for the most important metabolic BA receptor, TGR5, are typically low in both porcine and human peripheral circulation. Our findings raise questions about the magnitude of a peripheral TGR5 signal and its ultimate clinical application. 


\section{INTRODUCTION}

Bile acids (BAs) are intestinal lipid solubilizers, facilitating uptake of fats and fat-soluble vitamins. BAs are synthesised in the liver from cholesterol, conjugated to either glycine or taurine, and stored in the gallbladder. After a meal, BAs are released into the intestine and are taken back to the liver via the superior mesenteric and the portal veins, a very efficient BA transporter system (reviewed in (Dawson et al., 2009)). In the intestine, unconjugated BAs can diffuse passively over the intestinal border whereas conjugated forms require active transport to be taken up from the intestinal lumen. Active transport from the intestinal lumen is mediated by the apical sodium-dependent BA transporter (ASBT) in the distal ileum. Subsequently, BAs are transported over the basolateral side of the enterocyte via the organic solute transporter $\left(\mathrm{OST}_{\alpha} / \mathrm{OST}_{\beta}\right)$ and enter the liver via the portal vein. The liver clears most BAs from the portal blood via the $\mathrm{Na}^{+}$taurocholate cotransporting polypeptide (NTCP) which is highly expressed in the liver and has a high affinity for all conjugated BA. NTCP is aided by transporters of the organic anion transporting polypeptide (OATP) family that can transport unconjugated and sulphated BAs. In addition, it is hypothesized that the enzyme microsomal Epoxide Hydrolase (mEH) can also function as a sodium-dependent BA transporter on the sinusoidal plasma membrane (Kullak-Ublick et al., 2000). Hepatic BAs are excreted into the biliary tract via the bile salt export pump (BSEP) on the canalicular membrane.

Most of the BAs recycle in the enterohepatic circulation and only a small amount of BAs appear in the peripheral circulation. This may have important consequences for the presumed metabolic effects of BAs that are mediated by the nuclear farnesoid X receptor (FXR) and the transmembrane Takeda $\mathrm{G}$ protein-coupled receptor 5 (TGR5) (Kuipers et al., 2014). FXR regulates liver BA synthesis and metabolism, but can also contribute to glucose and lipid metabolism by stimulating the release of fibroblast growth factor (FGF) 15/19. TGR5 is not only proposed to mediate the effects of BAs on glucagon-like peptide-1 (GLP-1) and insulin release in the gut and pancreas, but also to affect energy 
expenditure and possibly insulin sensitivity via organs such as muscle or brown adipose tissue (Kuipers et al., 2014). Portal vein and peripheral plasma BA concentrations peak after a meal (Angelin and Bjorkhem, 1977; LaRusso et al., 1978; Schalm et al., 1978; Angelin et al., 1982). These observations suggest that BAs are potentially important postprandial signals to modulate metabolic and endocrine regulation in the gut, liver, muscle and fat.

Since the discovery of FXR and TGR5, it has been known that different BAs show a hierarchy in receptor activation (Parks et al., 1999; Maruyama et al., 2002; Kawamata et al., 2003; Sato et al., 2008). Additionally, transmembrane transport and conjugation may modulate activation patterns. Hence, porto-peripheral differences of different BA forms and their conjugation and hydrophobicity profiles may predict FXR and TGR5 activation in gut, liver and peripheral organs.

In an observational study, we investigated postprandial transhepatic BA fluxes in conscious pigs before and after a mixed meal (Figure 1). The pig model has been used for diabetes and metabolic research because of its resemblance to human physiology (Swindle et al., 2012). The aim of this study was to gain more insight in the postprandial transhepatic BA fluxes and plasma profiles of the different BAs and to predict the potential relevance of BAs for FXR and TGR5 activation. In addition, we analysed human portal and peripheral vein BA profiles to translate the experimental results to relevant human data. These data illustrate that the liver selectively extracts most BAs with high TGR5 affinity, which consequently are typically low in the peripheral circulation. 


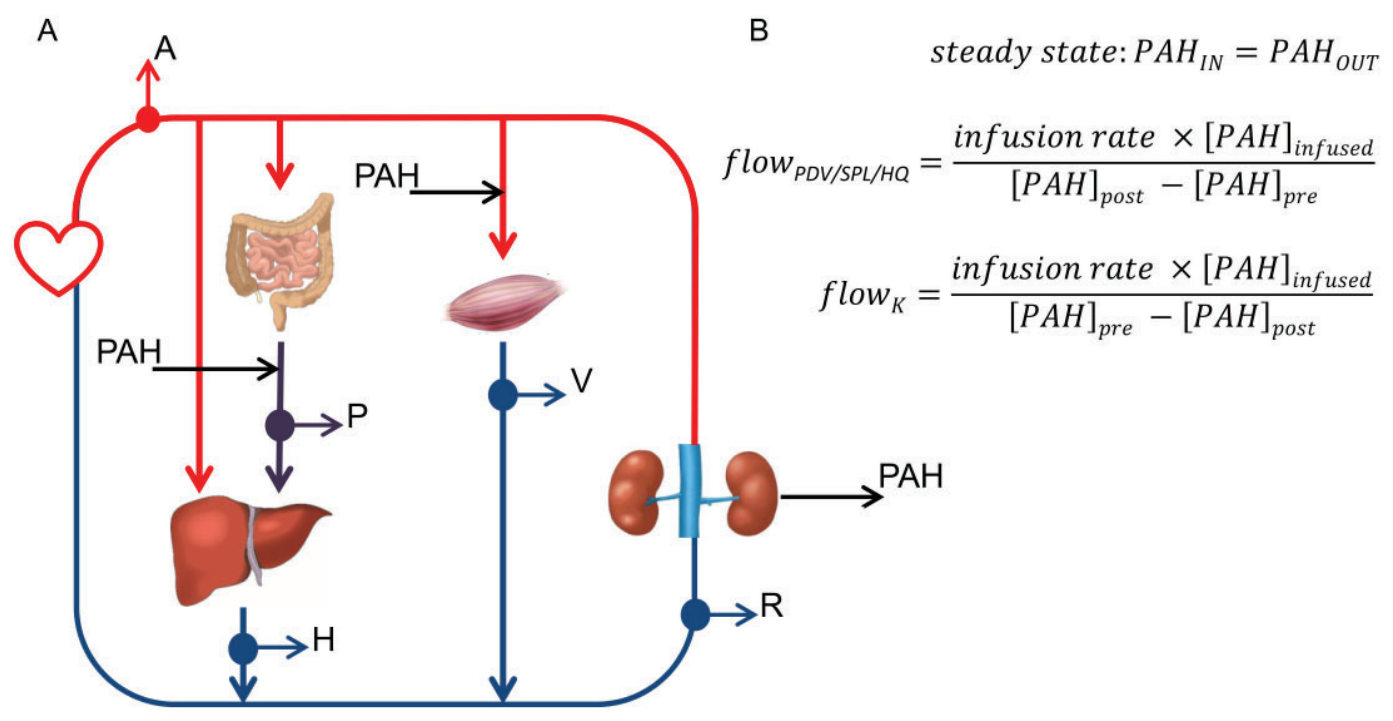

Figure 1. Intravascular sampling catheters and transorgan flux measurements.

(A) Schematic overview of placement of intravascular catheters. The blood stream is going in a clockwise direction from the heart with arterial blood in red and venous blood in blue. Circles with an arrow indicate sampling catheters and black arrows indicate the two infusion catheters for para-aminohippuric acid (PAH) and urinary loss via the kidneys. A: arterial line, sampling catheter in the abdominal aorta above the right renal artery to measure pre-organ concentrations; V: venous line, sampling catheter in the iliac circumflex profunda vein with its tip $5 \mathrm{~cm}$ above the bifurcation; R: renal line, sampling catheter in the left renal vein; P: portal line, sampling catheter in the portal vein with its tip in the liver hillus; $\mathrm{H}$ : hepatic line, sampling catheter in the hepatic vein by direct puncture of the liver. (B) Formulas to calculate the plasma flow through the organ compartments. Blood sampling started when $\mathrm{PAH}$ concentrations reached steady state (60 minutes). Plasma flow through the hindquarter muscle (HQ), the intestine (portal drained viscera, PDV) and the splanchnic compartment (SPL, intestine and liver) can be calculated with the upper flow formula. For $\mathrm{HQ}$ the PAH infusion site is the catheter in the abdominal aorta. For PDV and SPL the infusion site is the catheter into the splenic vein. $[\mathrm{PAH}]_{\text {pre }}$ is the $\mathrm{PAH}$ concentration in the main bloodstream, sampled through line $\mathrm{A}$. $[\mathrm{PAH}]_{\text {post }}$ is the $\mathrm{PAH}$ concentration in the efferent vein of the organ: line $\mathrm{P}$ for PDV, line $\mathrm{H}$ for SPL and line V for HQ. Plasma flow through the kidneys (K) can be calculated with the lower formula. Note that for $\mathrm{K}$ the extracted $\mathrm{PAH}$ is the infused $[\mathrm{PAH}]$ from both infusion sites. 


\section{MATERIALS AND METHODS}

\section{Animals}

Eleven female cross-bred pigs (20-25 kg, 8-12 weeks old) from a commercial breeder (Rosenbaum Farms, Brenham, TX) were individually housed in galvanized bar runs $(2 \times 3 \mathrm{~m})$ enriched with straw and toys to acclimatize for 2 weeks before surgical catheter placement. They were kept on a $12 \mathrm{~h}$ light-dark cycle (lights on at 7 AM) with a radio turned on during the light period. Environmental temperature was 21 $25{ }^{\circ} \mathrm{C}$. The pigs were fed $1 \mathrm{~kg} /$ day, Harlan-Teklad Vegetarian Pig/Sow Grower (Harlan laboratories, Indianapolis, IN). Water was available ad libitum. Data on acylcarnitines from this study have been published previously (Schooneman et al., 2015). All animal experiments were approved by the Institutional Animal Care and Use Committee of Texas A \& M University, USA.

\section{Surgery}

We placed the intravascular catheters one week before the experiment using the surgical techniques described earlier (Deutz et al., 1995; Ten Have et al., 1996). In short, we fasted the animals for $16 \mathrm{~h}$ before surgery and sedated them using an intramuscular injection of tiletamine-zolazepam $(3.3 \mathrm{mg} / \mathrm{kg})$ (Telazol; Zoetis, Kalamazoo, MI). Animals were intubated and the anaesthesia continued with isoflurane (2\%). In total, we implanted 7 intravascular catheters (Figure 1) as well as a feeding tube inserted percutaneously into the stomach.

In order to measure organ fluxes, we placed two catheters upstream of the organs for paraaminohippuric acid (PAH) infusion. For muscle flux measurements, we implanted the PAH infusion catheter into the abdominal aorta with its tip $5 \mathrm{~cm}$ above the bifurcation and the other PAH infusion catheter into the splenic vein. We inserted sampling catheters into the abdominal aorta above the right 
renal artery (A) for pre-organ compartment arterial plasma concentrations; into the inferior caval vein (iliac circumflex profunda vein) (V) with its tip $5 \mathrm{~cm}$ above the bifurcation for muscle flux measurements and venous concentrations; into the left renal vein $(R)$ for kidney flux measurements; into the portal vein with its tip in the liver hilus $(\mathrm{P})$, and into the hepatic vein $(\mathrm{H})$ by direct puncture of the liver for the splanchnic measurements. To reassure that the catheter $\mathrm{H}$ was correctly placed in the hepatic vein, we punctured the external side of the liver and pushed in the catheter for $20 \mathrm{~cm}$, then we retracted it to 5 $\mathrm{cm}$ to ensure we were not in the inferior caval vein. We secured all catheters in place and tunnelled them through the left abdominal wall. After abdominal closure the pigs were dressed with a canvas harness to protect the catheters. To keep the catheters patent they were filled with $0.5 \mathrm{~mL}$ of gentamycine $(20 \mathrm{mg} / \mathrm{mL})$ and $\alpha$-chymotrypsin solution $(225 \mathrm{U} / \mathrm{mL})$.

After surgery we checked the animals twice daily for four days on body temperature, catheter patency and overall behaviour. Also, animals received i.v. injections with antibiotics $(6.25 \mathrm{mg} / \mathrm{kg}$ lincomycin and $12.5 \mathrm{mg} / \mathrm{kg}$ spectinomycin) and analgetics ( $2 \mathrm{mg} / \mathrm{kg}$ flunixin meglumine) and the animals were allowed to recover for 7-10 days. During this period they were also habituated to the experimental cage $(0.9 \times 0.5 \times 0.3 \mathrm{~m}$ on wheels).

\section{Experimental procedure}

The animals were conscious during the whole experimental procedure. We removed all food at 16:00h the day before the experiment. On the day of the experiment all animals were first weighed and at 08:00 $\mathrm{h}(\mathrm{t}=-60 \mathrm{~min})$, an hour before the meal, we started the continuous infusion of $25 \mathrm{mM} \mathrm{PAH}$ at $60 \mathrm{~mL} / \mathrm{h}$ and reached steady state before the sampling started. At $t=-10,-5$ and $0 \mathrm{~min}$, we took three baseline blood samples from all catheters. Samples were always taken in the same order during the experiment: A, $P, H, V, R$. At 09:00 h, t = 0, the pigs received their test meal via the gastric feeding tube. For a pig of 25 
$\mathrm{kg}$, the test meal consisted a $600 \mathrm{~mL}$ mixture of $78 \mathrm{~g}$ of crude whey protein isolate $(100 \%$ Premium Whey Protein; Body Fortress, Bohemia, NY) and $110 \mathrm{~g}$ of carbohydrates (Malto dextrin; Muscle Feast, Hebron, $\mathrm{OH}$ ) in water, and $22 \mathrm{~g}$ of olive oil (30\% of daily energy intake; Korger, Cincinnati, OH). We administered the complete test meal within approximately 5 min and took postprandial blood samples at $t=10,20,30,45,60,90,120,180$ and 240 min. After the experiment, pigs were returned to their normal cages with their normal food and water available. Malfunctioning catheters accounted for missing data (two hepatic vein catheters and one renal vein catheter).

\section{Sample preparation}

After withdrawal, we immediately placed the blood samples on ice. For the PAH concentration measurements, we pipetted $250 \mu \mathrm{L}$ of blood into a tube containing $25 \mu \mathrm{L}$ of trichloroacetic acid (TCA), thoroughly vortexed, and subsequently, together with the remaining blood samples, centrifuged at 8000 g for 5 min at $4{ }^{\circ} \mathrm{C}$. After spinning, we transferred the plasma samples into clean tubes and snap froze them in liquid nitrogen and stored them at $-80{ }^{\circ} \mathrm{C}$ until further analysis. For measurements, samples were defrosted and thoroughly homogenized using a multivortex and subsequently centrifuged for $3 \mathrm{~min}$ at 1780 or $1800 \times \mathrm{g}$ and aliquoted for BA, insulin and glucose concentration measurements.

\section{PAH concentrations and flow calculations}

The PAH concentrations of the TCA plasma samples were compared to PAH standards and read out using a microplate spectrophotometer (Spectramax; Molecular Devices, Sunnyvale, CA) and SoftmaxPro software (Molecular Devices) (Agarwal, 2002). We calculated plasma flow through the organs using the dilution of PAH over the organ compartment (Deutz et al., 1995; Ten Have et al., 1996). Blood sampling 
(Figure 1) started when PAH concentrations reached steady state (60 minutes). So $\mathrm{PAH}_{\mathrm{IN}}=\mathrm{PAH}_{\mathrm{OUT}}$. We calculated the plasma flow through the hindquarter muscle $(\mathrm{HQ})$, the intestine (portal drained viscera, PDV) and the splanchnic compartment (PDV and liver, SPL) with the formula: flow $=\frac{\text { infusion rate } \times[P A H]_{\text {infused }}}{\left[{ }^{[P H}\right]_{\text {post }}-[P A H]_{\text {pre }}}$. For $\mathrm{HQ}$ the PAH infusion site is the catheter in the abdominal aorta. For PDV and SPL the infusion site is the catheter into the splenic vein. $[\mathrm{PAH}]_{\text {pre }}$ is the PAH concentration in the main bloodstream, sampled through line $\mathrm{A} .[\mathrm{PAH}]_{\text {post }}$ is the PAH concentration in the efferent vein of the organ: line P for PDV, line $\mathrm{H}$ for SPL and line $\mathrm{V}$ for HQ. Plasma flow through the kidneys $(\mathrm{K})$ can be

calculated with the formula: flow $_{k}=\frac{\text { infusion rate } \times[P A H]_{\text {infused }}}{\left.{ }^{[P A H}\right]_{\text {pre }}-[P A H] \text { post }}$. Note that for $\mathrm{K}$ the extracted PAH is the infused $[\mathrm{PAH}]$ from both infusion sites. We interpolated missing data points and used the mean plasma flow of all pigs for further flux calculations.

\section{Glucose and insulin concentrations}

We measured glucose concentrations in duplicate with a glucose oxidase method using the Biosen C-line plus glucose analyser (EKF Diagnostics, Barleben/ Magendeburg, Germany) and insulin concentrations in duplicate with a porcine Insulin ELISA (version 4.0, Mercodia, Uppsala, Sweden) according to the manufacturer protocol. Plates were read with a spectrophotometer (Varioskan Flash version 2.4.3, Thermo Scientific) running matching Skanlt software. The calibration curve was a cubic polynomial extrapolated to concentrations of 0.01 to $2.0 \mathrm{ng} / \mathrm{L}$. Samples outside this range were set to $\leq 0.01$ or $\geq 2.0$ $\mathrm{ng} / \mathrm{L}$, respectively. Insulin concentrations were not measured in renal vein samples. 


\section{BA concentrations}

We measured BA concentrations by liquid chromatography-tandem mass spectrometry (LC/MS/MS, Supplemental Methods). Pigs have an abundant BA profile (Legrand-Defretin et al., 1991; Ferezou et al., 1997). Here, we focused mainly on BAs that are prevalent in humans to enable translation: ursodeoxycholic acid (UDCA), cholic acid (CA), chenodeoxycholic acid (CDCA), deoxycholic acid (DCA), lithocholic acid (LCA) and hyodeoxycholic acid (HDCA, non-human), and their glycine- (g) and taurine- (t) conjugated forms. In the humans, 15 BAs were measured: UDCA, CA, CDCA, DCA and LCA and their gand t-conjugated forms. For sample preparation, after homogenizing and spinning, $25 \mu \mathrm{L}$ plasma was aliquoted into a clean tube for BA analysis. For every 10 samples prepared, one quality control standard plasma was included. To each sample, we added $250 \mu \mathrm{L}$ internal standard and vortexed for $60 \mathrm{~s}$. Samples were centrifuged at $15800 \times \mathrm{g}$ and the supernatant poured into a clean glass tube. The fluid was

evaporated under nitrogen at $40{ }^{\circ} \mathrm{C}$. Before measuring samples were reconstituted in $100 \mu \mathrm{L} 50 \%$ methanol in water, vortexed for $60 \mathrm{~s}$ and centrifuged for $3 \mathrm{~min}$ at $1800 \times \mathrm{g}$. We transferred the supernatant into a $0.2 \mu \mathrm{m}$ spin-filter and centrifuged at 2,000 x g for $10 \mathrm{~min}$. After filtering, the samples were transferred into $\mathrm{LC} / \mathrm{MS}$ vials and analysed (10 $\mu \mathrm{L}$ injection volume). The lower limit of quantification (LOQ) was $0.05 \mu \mathrm{M}$ for all BA forms.

In pigs, all measured concentrations of CA, tCA, gCA, DCA, gDCA and tDCA were below the LOQ. Unconjugated UDCA concentration was never calculated due to interfering peaks in the chromatogram. Therefore, we excluded the conjugated and unconjugated forms of CA and DCA, and unconjugated UDCA from analysis. In humans, UDCA, tUDCA and tDCA were excluded from analysis due to interfering peaks in the chromatogram. For all the other BAs, when a concentration was below the lower LOQ, its value was set to $50 \%$ of the detection limit i.e., $0.025 \mu \mathrm{M}$. 


\section{Organ flux calculations}

In the present model we measured organ flux through the hindquarter muscle compartment (muscle flux), the kidney compartment (renal flux), the intestine and pancreas compartment (portal drained viscera (PDV) flux) and the splanchnic compartment: the PDV plus the liver (SPL flux). We calculated liver flux as SPL flux minus PDV flux. Flux through a compartment was calculated as the mean plasma flow through that compartment multiplied with the arterio-venous difference in concentration of glucose, insulin or BA. A positive flux value is interpreted as appearance or production by the compartment, while a negative flux is interpreted as disappearance or uptake in the compartment.

\section{Human study}

We took blood samples from the cubital vein and portal vein from consecutive subjects during elective gastric by-pass surgery. Eleven healthy non-diabetic patients participated in this study (1 male, 10 females; age $46.0 \pm 12.7 \mathrm{yrs}$; body mass index $41.4 \pm 2.8 \mathrm{~kg} / \mathrm{m}^{2}$; mean $\pm \mathrm{SD}$ ). All participants gave informed consent before the surgery and the protocol was approved by the Medical Ethical Committee

of the Academic Medical Centre and Slotervaart Hospital, Amsterdam. None of the patients used BA sequestrants. Subjects were fasted overnight prior to the surgery. First, a pre-operative peripheral venous sample was taken. During the surgery, the portal vein puncture was performed as previously described (Fontana et al., 2007). Glucose, insulin and BA concentrations were measured in these samples. 


\section{Statistics}

Calculations were made using Microsoft Excel 2010 version 14.0 and area-under-the-curve analysis and statistics were done in Graphpad Prism version 7. For porcine data analysis paired t-test or repeated measure ANOVA with Tukey post-hoc analysis were used where appropriate. For human data we used non-parametric tests. Detected numeric outliers were excluded based on Grubbs' test. Data are presented as mean \pm standard error of the mean (SEM).

\section{RESULTS}

\section{Glucose and insulin concentrations and transorgan fluxes validate the porcine model}

In addition to the BAs, we measured glucose and insulin concentrations and transorgan fluxes. These data confirm the reproducibility of our postprandial model. The postprandial responses of glucose and insulin concentrations showed similar responses in all pigs with low interindividual variability (Supplemental Figures S1-4). Plasma glucose and insulin concentrations increased after the meal in all blood vessels as shown in Figure 2A, B. Postprandial concentrations of both glucose and insulin peaked within the first hour and, subsequently, returned to baseline in 4 hours. As expected, the concentrations of both glucose and insulin were highest in the portal vein, since glucose and insulin directly appear here postprandial from the intestine and pancreas, respectively (AUC glucose mmol/L one-way ANOVA: portal $2143 \pm 90$ vs hepatic $1744 \pm 119$, arterial $1727 \pm 85$, renal $1629 \pm 92$ and venous $1571 \pm 80 ; p<0.0001$ AUC insulin $\mu \mathrm{mol} /$ L one-way ANOVA: portal $162 \pm 14$ vs hepatic $104 \pm 12$, arterial $88 \pm 8$ and venous: $81 \pm$ 9; $p<0.0001$ ). Figure $2 C, D$ show that glucose and insulin transorgan fluxes in the fasted state (i.e., $t=0$ ) were low, except for the positive liver flux, which reflects hepatic glucose output. Transhepatic glucose flux increased initially, but subsequently decreased to zero within one hour. Therefore, after 
administration of the meal, hepatic glucose production may have continued for a brief period. Together with glucose from the portal drained viscera (PDV, i.e., intestine and pancreas), this resulted in a substantial postprandial glucose peak in the peripheral circulation. Insulin flux across the liver was negative which demonstrates hepatic insulin clearance. As a consequence, plasma insulin concentrations in the hepatic vein and beyond were substantially lower than in the portal vein. The postprandial negative muscle flux indicates uptake of glucose by the muscle. The negative kidney glucose flux indicates uptake of glucose by the kidneys.

\section{BAs continuously cycle in the enterohepatic circulation of pigs regardless of food status}

Total BA concentrations in the portal vein were at least 6 times higher than in the other blood vessels, reflecting that most BAs are contained within the enterohepatic cycle (fasted state total BA concentration in portal vein: $25.01 \pm 2.8 \mu \mathrm{M}$ and caval vein: $4.29 \pm 1.0 \mu \mathrm{M}, \mathrm{p}<0.0001)$. In addition, the baseline flux of portal vein BAs indicates that BAs also circulate in the enterohepatic circulation in the postabsorptive state, while total BA concentrations between the hepatic, renal or caval veins and aorta were not different (Figure 3B-E). 

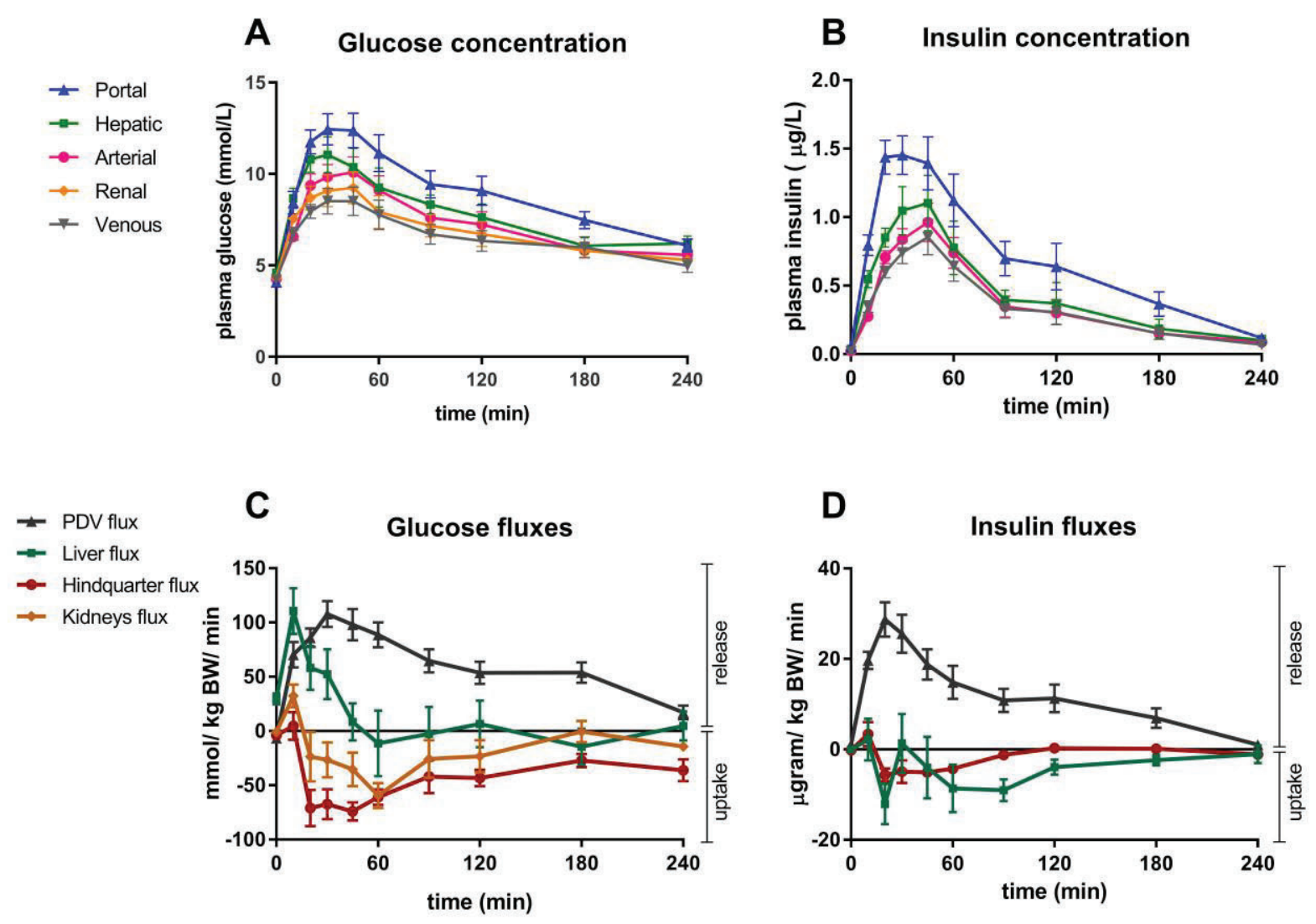

Figure 2. The postprandial glucose and insulin response throughout the body.

Postprandial plasma concentrations (A-B) and transorgan fluxes (C-D) for glucose (A, C) and insulin (B, D). Fasted state before the meal is averaged at $t=0$. A positive flux denotes net production by the organ(s). A negative flux reflects net uptake by the organ(s). Please note that in the fasted state liver glucose flux is positive $(30 \mathrm{mmol} / \mathrm{kg}$ $\mathrm{BW} / \mathrm{min}$ ). Data represented as mean \pm SEM. PDV: portal drained viscera (intestine and pancreas). $\mathrm{N}=11$, except for the hepatic line $(\mathrm{N}=9)$ and renal line $(\mathrm{N}=10)$ and appurtenant analysis. 
A

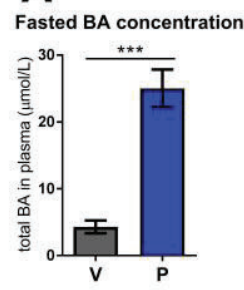

B

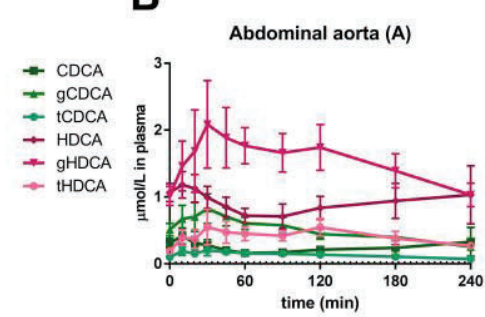

C

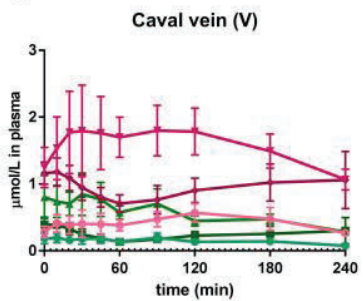

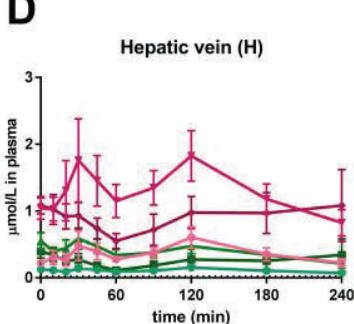

E

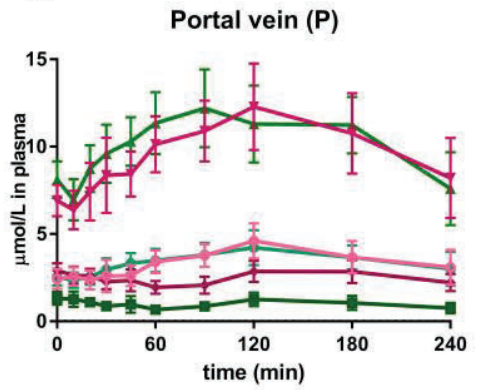

F bile acid concentration-time curves

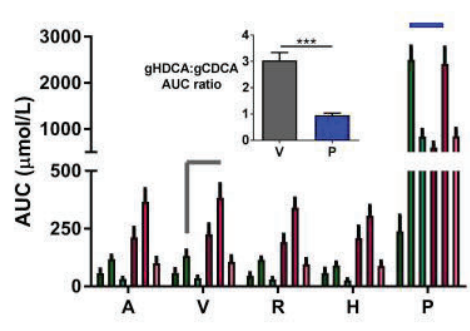

G

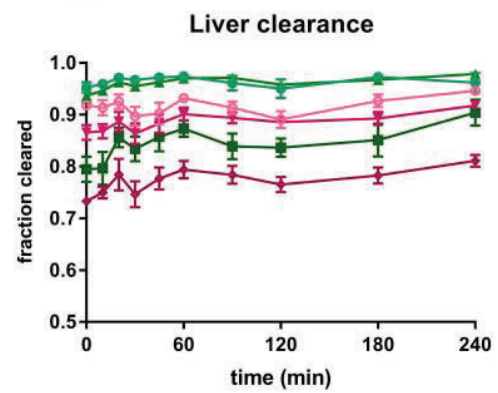

Figure 3. Postprandial released BAs are efficiently cleared by the liver.

(A) During fasting enterohepatic BA concentrations $(P)$ are $\sim 6$ times higher than peripherally (V). (B-E) BA concentration curves show an increase after food intake, time 0 represents the fasted state. CDCA: chenodeoxycholic acid (green), HDCA: hyodeoxycholic acid (pink), g: glycine (triangle), t: taurine (circle). (F) The individually depicted postprandial area under the curve (AUC) of CDCA and HDCA and their conjugates shows that the main difference between the enterohepatic and peripheral BA profile is the ratio between gCDCA and gHDCA. Peripheral BA profiles are very similar. R: renal line. (G) Hepatic uptake of conjugated BA is higher than for unconjugated forms and especially conjugated CDCA is efficiently cleared. BA clearance was calculated as [BA] (portal vein + aorta) $-[B A]$ hepatic vein $/[B A]$ (portal vein + aorta). Data represented as mean $\pm \mathrm{SEM} . \mathrm{N}=11 ;$ hepatic outcomes $(\mathrm{N}=9) .{ }^{* * *} \mathrm{p}<0.001$, two-tailed paired t-test. 


\section{Postprandial BA concentrations rise with large variability between animals}

After the meal, BA concentrations increased in the portal vein, and, to a much lesser extent, in the peripheral circulation, with a broad peak around 1.5 to 2 hours after the meal (Figure 3B-E, Supplemental Figure S10). In contrast to the relatively uniform postprandial glucose and insulin responses, these postprandial BA responses revealed relatively large inter-animal variabilities (Supplemental Figures S5-9). When aligning the individual postprandial concentration curves at their peak a significant postprandial rise in BA concentration becomes apparent, for example for glycineconjugated hyodeoxycholic acid (gHDCA) (Supplemental Figure S11). The most prominent BAs present in the portal vein were glycine-conjugated (g) chenodeoxycholic acid (CDCA) and gHDCA, followed by their taurine-conjugated $(\mathrm{t}$ ) and unconjugated forms (Figure 3E,F). Conjugated and unconjugated forms of lithocholic acid (LCA) and ursodeoxycholic acid (UDCA) were well detected in the portal vein but their concentrations were very low elsewhere (Figure 4).

\section{Porcine hepatic clearance is most efficient for conjugated CDCA forms}

The BA profile in the peripheral circulation was similar to the portal vein, albeit with much lower concentrations except for gCDCA (Figure 3 and Figure 4). Even though gCDCA and gHDCA were the most prominent BAs in the portal vein, hepatic vein concentrations of gCDCA were much lower than gHDCA concentrations (Figure 3F; ratio gHDCA:gCDCA in P: $0.95 \pm 0.1$ vs in V: $3.04 \pm 0.3 ; p<0.0001$ ). To quantify hepatic clearance rates, we calculated the differences between BA concentrations proximal and distal of

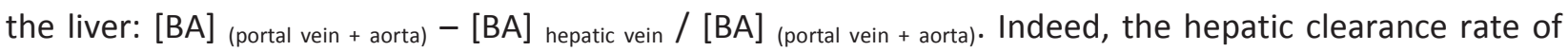
gCDCA was significantly higher than that of gHDCA ( $t=60 \mathrm{~min}, \mathrm{p}<0.0001$, Figure $3 \mathrm{G}$ ). In general, the hepatic clearance rates of conjugated BA were significantly higher than the clearance rates of their unconjugated forms. 

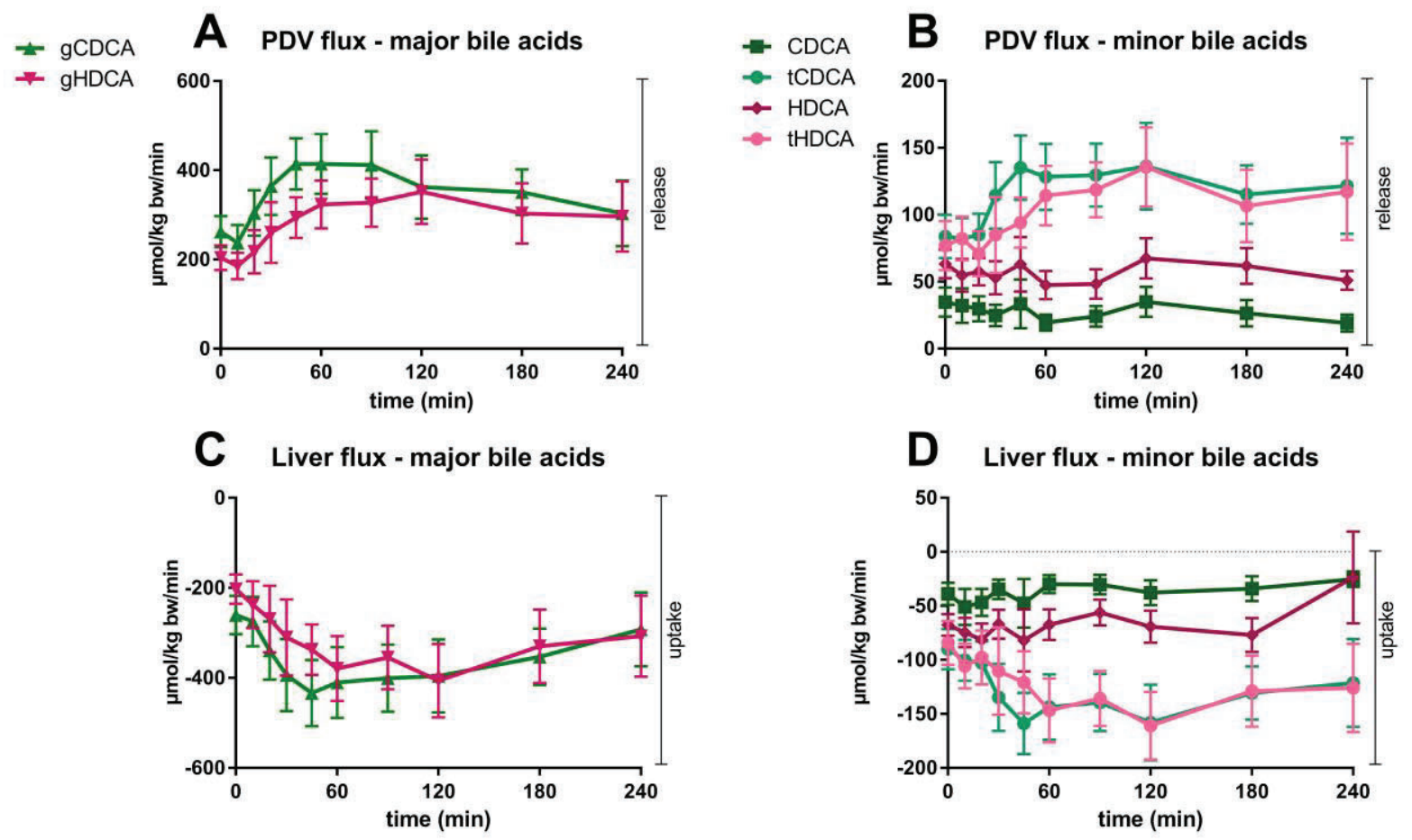

Figure 4. Lithocholic acid forms, BAs with high TGR5 affinity, are mainly found in the enterohepatic circulation and not peripherally.

(A) Lithocholic acid (LCA) and its glycine- $(\mathrm{g})$ and taurine- $(\mathrm{t})$ conjugated forms are present in pig plasma in stable low concentrations. gLCA shows a postprandial increase in the portal vein but not in the peripheral circulation. The other forms do not show a postprandial response and conjugated forms of LCA are not even detected outside the enterohepatic circulation. Fasted state: $\mathrm{x}=0$, PDV: portal drained viscera, dotted line indicates the $50 \%$ detection limit, undetected concentrations were set to this number $(0.025 \mathrm{mmol} / \mathrm{L}) . \mathrm{N}=11$. (B) Transorgan fluxes of LCA forms. Like the other BAs LCA and conjugates are released from the intestine and very efficiently taken up by the liver. 


\section{Postprandial BA exposure is high in the enterohepatic cycle compared to the peripheral circulation}

Transorgan flux is different from clearance, since it is calculated by multiplying concentration difference by plasma flow. In the fasted state, BA flux in the portal vein was considerable (i.e., enterohepatic cycle). However, after the meal transorgan fluxes doubled, in particular of the glycine-conjugated BA (Figure 5A). Thus, the postprandial exposure to BA in the enterohepatic circulation was high. The postprandial peak in portal BA concentrations was also clearly visible in the BA fluxes, as shown in Figure 5. For all BAs the PDV flux was always positive with a clear postprandial peak, while the liver flux was negative and mirrored the curve of the PDV flux. These fluxes show the appearance of BA from the gut into the portal vein and the efficient absorption of BA by the liver. There was no net transorgan BAs flux over the kidneys and hindquarter (Supplemental Figure S11).

\section{Peripheral exposure to the secondary BA with the highest TGR5 affinity is low}

Lithocholic acid (LCA) is a secondary BA that has a high affinity for TGR5. Therefore, LCA had our special interest. In the fasted state unconjugated LCA concentration in the portal vein was approximately two times higher than gLCA and tLCA (Figure 4A). In general, LCA and its conjugates were detected in all portal samples, but not in all samples from other sampling sites. After the meal, portal gLCA concentration showed a robust postprandial peak, in contrast to LCA and LCCA concentrations that did not change compared to the fasted state (Figure 4A). PDV and liver flux curves resembled the postprandial concentration curves, although, because of the biliary secretion, the liver flux was negative (Figure 4B). Therefore, analogous to primary BAs, the glycine-conjugated forms increase after the ingestion of a meal. In the peripheral circulation LCA concentration was five times lower compared to the portal vein (Figure 4A, $p<0.01$ ). Conjugated forms of LCA were undetectable. 


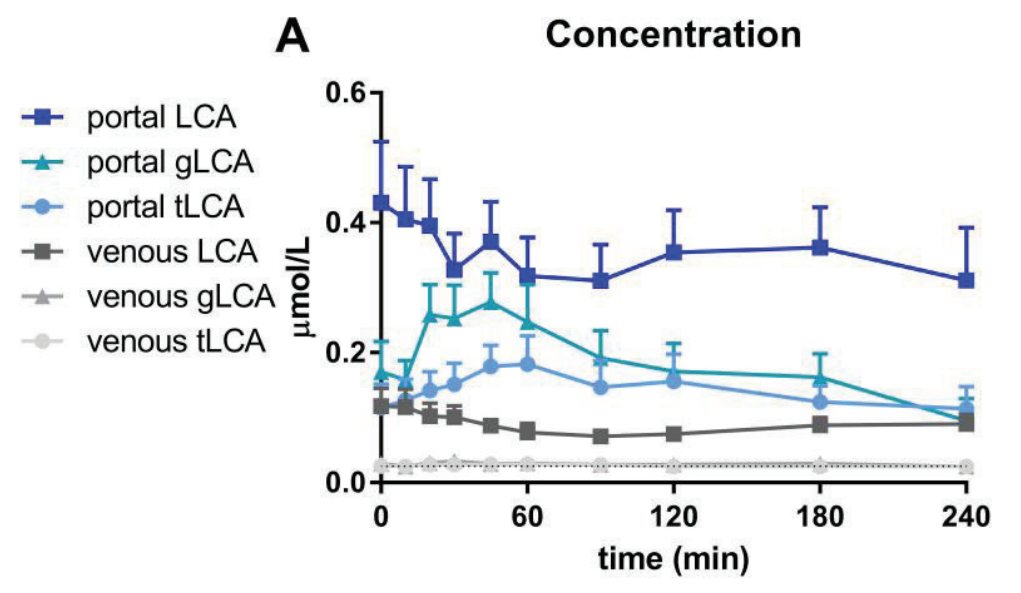

B
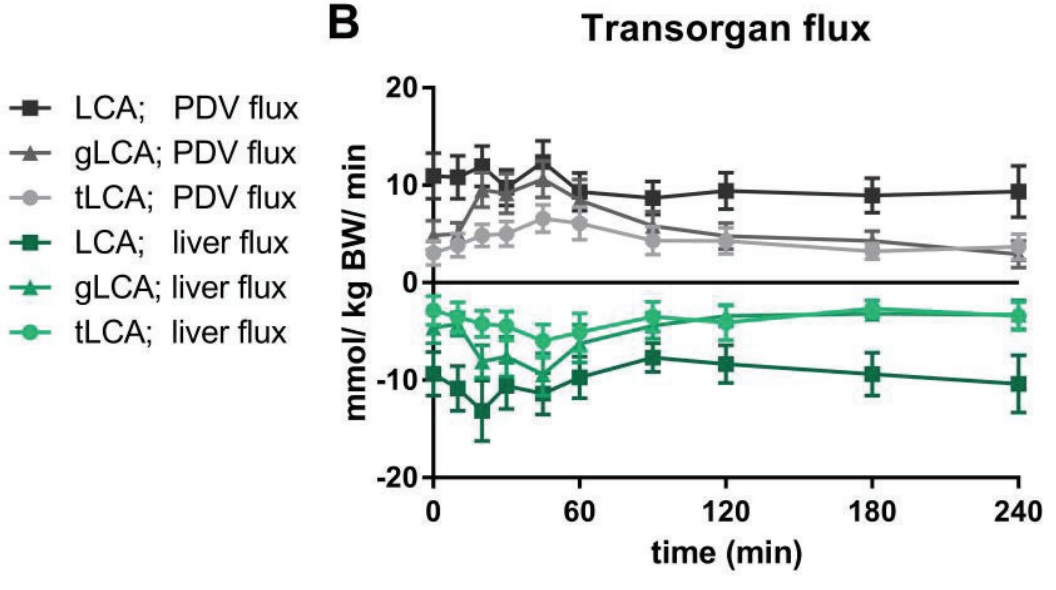

Figure 5. BAs are released from the intestine and subsequently very efficiently absorbed by the liver.

(A and B) After meal intake the BA flux over the portal drained viscera (PDV) steeply increases. A positive flux denotes net production by the organs. Data represent mean \pm SEM with $N=11$. ( $C$ and $D$ ) Postprandially the liver takes up most released BAs. A negative flux reflects net uptake by the organ. CDCA: chenodeoxycholic acid, HDCA: hyodeoxycholic acid, g: glycine-conjugated form, t: taurine-conjugated form. Data represent mean \pm SEM with $\mathrm{N}=$ 9. 


\section{Translating the porcine BA profile to humans}

We translated our porcine findings to humans using portal and peripheral blood samples of patients during bariatric surgery. All subjects were obese, but none of the subjects had type 2 diabetes mellitus (mean \pm standard deviation: fasting plasma glucose: $5.6 \pm 0.5 \mathrm{mmol} / \mathrm{L}$; fasting plasma insulin: $73 \pm 31$ pmol/L). Total BA concentrations were significantly higher in the portal vein compared to the peripheral circulation: $7.43 \pm 7.2$ vs $0.68 \pm 0.5 \mu \mathrm{mol} / \mathrm{L}$, respectively $(p=0.01)$. In peripheral veins $B A$ concentrations were low and consisted of conjugated and unconjugated forms (Figure 6). Portal BA concentrations were high and consisted mainly of conjugated forms, whereas the concentrations of unconjugated BAs were similar to those in the periphery (Figure 6). Similar to pigs, LCA and conjugates were only detected in the portal vein. However, the human BA profile contained another secondary BA, i.e., deoxycholic acid (DCA), that circulated in the portal vein and the periphery and has been associated to insulin sensitivity (Brufau et al., 2010; Cariou et al., 2011; Haeusler et al., 2013).

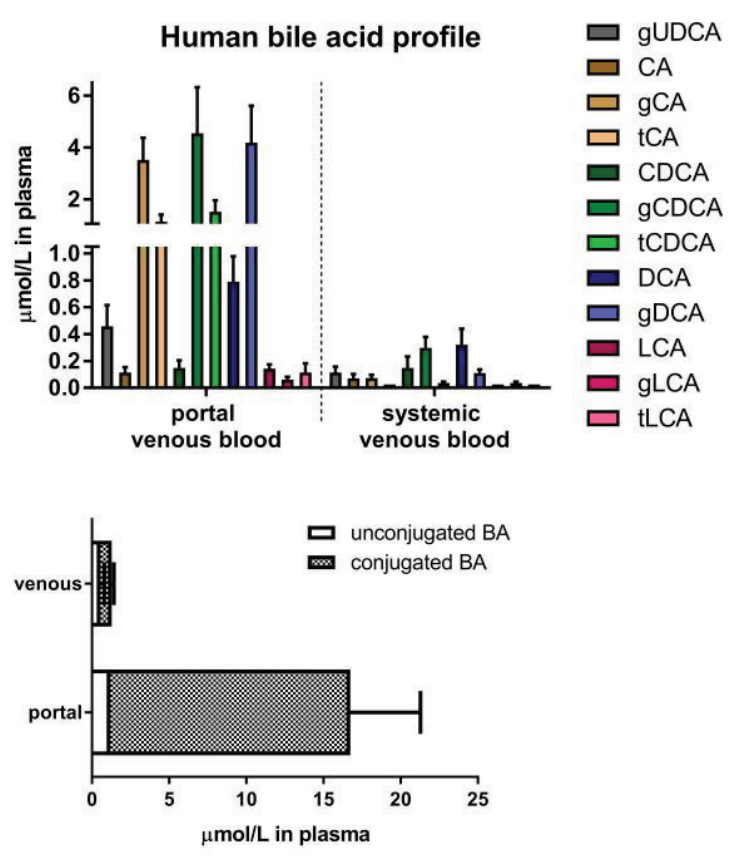

Figure 6. Portal and peripheral plasma BA profile of nondiabetic obese humans.

Portal BA concentrations are much higher than peripheral plasma concentrations. In humans most of the BAs in plasma are conjugated to glycine. UDCA: ursodeoxycholic acid, CA: cholic acid, CDCA: chenodeoxycholic acid, DCA: deoxycholic acid, LCA: lithocholic acid, g: glycine-conjugated, t: taurineconjugated. Data represented as mean \pm SEM. $\mathrm{N}=11$. 


\section{DISCUSSION}

The aim of this study was to quantify porto-peripheral postprandial plasma BA profiles and transorgan flux. Fasting and postprandial peripheral and portal BA concentrations and fluxes are substantial which is supported by the enterohepatic cycle turnover of 12 times per day (Mok et al., 1977; Lefebvre et al., 2009). Physiological cycling of the BA pool may have advantages. In general, metabolic or endocrine cycles enable rapid physiological adaptations when demanded. In the case of the enterohepatic cycle, continuous cycling permits a swift increase of BAs (i.e., concentration) when needed postprandially. Continuous cycling of BA may also prevent unlimited BA synthesis via FGF15/19 effects on CYP7A1, which is the rate-limiting enzyme of the major classical pathway in BA biosynthesis (Holt et al., 2003; Inagaki et al., 2005). Liver and intestinal FXR KO murine models have shown that Cyp7a1 repression depends mainly on intestinal FXR activation via FGF15 (Kim et al., 2007; Kong et al., 2012). Additionally, BAs inhibit Cyp7a1 via FXR and small heterodimer partner (SHP) in the liver (Goodwin et al., 2000; Lu et al., 2000; Kuipers et al., 2014).

The postprandial BA curves showed a large variability. La Russo et al. found that in humans peripheral conjugated CA forms show modest intra-individual variability with respect to the time of peak, however, peak height in these subjects showed up to $30 \%$ variation (LaRusso et al., 1978). Steiner et al. describe in detail the intra- and inter-individual variability in daily peripheral plasma BA concentrations of 4 healthy humans (Steiner et al., 2011). Intra-individual variability of postprandial BA curves and its determinants (e.g., gut luminal BA appearance, gut microbioma characteristics, BA transporter genotypes, BA synthetic capacity, gut motility and others) need to be investigated in future studies.

We focused on human BAs and the porcine BA profile mainly consists of CDCA and HDCA forms. CDCA is a primary BA, whereas HDCA may be both primary (since it was detected in germ free pigs (Haslewood, 1971)) and secondary. Notably, the concentration of the unconjugated forms was 
unaffected by the ingestion of a meal. So the postprandial response was mainly explained by the glycineconjugated forms of CDCA and HDCA. Differences in the postprandial response between conjugated and unconjugated BAs may be due to microbiota and the affinity of the BA forms for their transporters (Dawson et al., 2009). We found that conjugated BAs are more efficiently cleared than their unconjugated forms, showing the high effectiveness and expression of NTCP in the liver. HDCA cannot diffuse passively and is only a substrate for OATP1, but not for NTCP, which might explain the relative lower HDCA clearance compared to CDCA forms (Hata et al., 2003). Despite the large increase in conjugated BA flux through the enterohepatic pathway after food intake, hepatic clearance of the BAs remained stable. The unconjugated BAs flow through the enterohepatic cycle passively regardless of food status and do not appear to have an additional role in postprandial fat digestion (Hofmann, 1999).

LCA forms have the highest TGR5 affinity, but are often ignored in humans because of their low peripheral plasma concentrations. In the pigs, we showed that unconjugated LCA outnumbered conjugated LCA forms in contrast to HDCA and CDCA forms. The unconjugated LCA can either be derived from deconjugation and transformation of CDCA, or deconjugation of gLCA or tLCA by gut bacteria (Hofmann and Hagey, 2008). LCA was already high in fasted samples and did not show a postprandial change, again indicating ongoing cycling and deconjugation without food intake. However, gLCA showed an increase after the meal, which is likely to rely on early re-uptake of gLCA that has been excreted by the liver. The human LCA conjugation profile was very much comparable to the porcine profile enabling translation. In the enterohepatic circulation the LCA forms and concentrations are likely sufficient to signal TGR5 in intestinal L-cells or liver Kupffer cells.

The porcine BA forms, i.e., HDCA, are not found in humans, whereas pigs do not have DCA which has a relative high TGR5 affinity (Sato et al., 2008). To speculate on the potential of the plasma bile acid pool to activate TGR5 we used a simplified method to calculate the hypothetical TGR5 activating capacity 
for which we used the plasma BA concentration and the published $\mathrm{EC}_{50}$ of the BAs for TGR5 (Figure 7) (Sato et al., 2008). This superficial approach does not take into account the shape and range of linearity of the concentration-activation curve and the resulting measure overestimates TGR5 activity since BA concentrations were below their $\mathrm{EC}_{50}$ value. However, it shows that TGR5 activation potential may be much higher in the portal vein than elsewhere, most likely because concentrations are also much higher in the portal vein. Interestingly, calculated human TGR5 activation capacities were not different from pigs, whereas the pool composition is very different. This could be explained by the fact that pigs had higher concentrations of BAs, including LCA, and thus similar TGR5 signalling.

Postprandial BAs have been studied to a limited extend, however this is the most sizable study so far in terms of postprandial time points, number of animals/humans and number of individual LC/MS/MS BA analyses (Angelin et al., 1982). We did not perform activating calculations for FXR since the necessary data are not uniformly published for this receptor (Makishima et al., 1999; Parks et al., 1999). Alternatively, a more uniform hydrophobicity index has been used to qualify BAs, but these data are not available for all BAs (Heuman, 1989; Roda et al., 1990; Twisk et al., 1995). In addition, FXR is located in the nucleus, which complicates the calculation of potential FXR activation with plasma BA concentrations even more.

Our study has strengths and weaknesses. The porcine model is suitable for diabetes and metabolic research because it mimics human physiology and pathophysiology in many respects (Swindle et al., 2012). More importantly, the pig has a day-night rhythm comparable to humans, is omnivorous and its intestinal transit time and efficiency of digestion resemble that of humans (Miller and Ullrey, 1987). In an effort to translate the porcine data to humans, we included healthy obese subjects undergoing bariatric surgery. Fasting plasma BA concentrations are not different in obese subjects despite lower postprandial concentrations(Glicksman et al., 2010; Haeusler et al., 2016). BMI is positively 
correlated to total BA concentrations and markers for hepatic BA synthesis in plasma (Prinz et al., 2015; Haeusler et al., 2016). So the human data presented in this study may alter from (postprandial) BA metabolism in lean subjects. Our speculation that TGR5 activation is greater within the entero-hepatic cycle would greatly benefit from additional measurements in the pigs such as plasma GLP-1 concentrations since intestinal TGR5 activation triggers release of GLP-1 (Jain et al., 2012). In addition, FGF19 concentrations would be interesting to indicate activation of the FXR-FGF19 axis and its beneficial effects on liver metabolism (Jain et al., 2012). Unfortunately, the numerous blood samples limited the amount of blood per sample. Feeding pigs via a gastric tube may have prevented a normal postprandial hedonic response, but the amount and composition of the meal still elicited a robust physiological postprandial response. Finally, animals received antimicrobial therapy peri-operatively and this may have affected BA pool composition although this has not been reported previously for the antibiotics that we used.

In conclusion, the liver selectively extracts most BAs and BAs with high TGR5 affinity are typically low in the porcine and human peripheral circulation. Our data do not preclude a role for BAs to activate TGR5 in the periphery postprandially. However, their low peripheral plasma concentrations and high interindividual variability raise questions about the magnitude of such a signal and the ultimate clinical application of TGR5 agonists if the liver is not passed (van Nierop et al., 2017). 


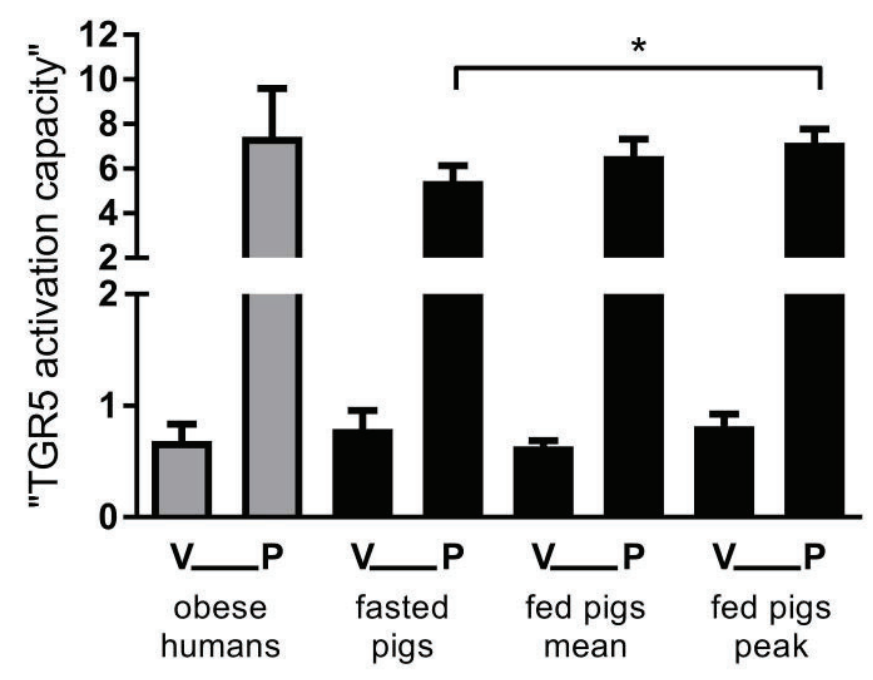

Figure 7. Hypothetical TGR5 activating capacity is higher for the portal vein compared to the periphery, independently of food intake.

This measure expresses in one number the potential amount of TGR5 activation by the total bile acid pool and is calculated for individual BAs in the pool by dividing the average concentration by their $\mathrm{EC}_{50}$, whereafter all are totalled. The value is lower in a peripheral vein (V) compared to the portal vein (P) in healthy obese humans (grey) and in pigs (black). There are no differences between the fasted and the postprandial state (fed pigs vs fasted pigs). However, when comparing the fasted state to the peak of the postprandial concentration curve (fed pigs peak) food intake caused a significant increase in the TGR5 activating potential of over $30 \%$. Data represent mean \pm SEM. Porcine outcomes fasted vs fed staged were tested with a one-way ANOVA and Tukey post-hoc test, $* p<0.05$. 


\section{REFERENCES}

Agarwal, R. (2002). Rapid microplate method for PAH estimation. Am J Physiol Renal Physiol 283(2), F236-241. doi: 10.1152/ajprenal.00336.2001.

Angelin, B., and Bjorkhem, I. (1977). Postprandial serum bile acids in healthy man. Evidence for differences in absorptive pattern between individual bile acids. Gut 18(8), 606-609.

Angelin, B., Bjorkhem, I., Einarsson, K., and Ewerth, S. (1982). Hepatic uptake of bile acids in man. Fasting and postprandial concentrations of individual bile acids in portal venous and systemic blood serum. J Clin Invest 70(4), 724-731.

Brufau, G., Bahr, M.J., Staels, B., Claudel, T., Ockenga, J., Boker, K.H., et al. (2010). Plasma bile acids are not associated with energy metabolism in humans. Nutr Metab (Lond) 7, 73. doi: 10.1186/1743-7075-7-73.

Cariou, B., Chetiveaux, M., Zair, Y., Pouteau, E., Disse, E., Guyomarc'h-Delasalle, B., et al. (2011). Fasting plasma chenodeoxycholic acid and cholic acid concentrations are inversely correlated with insulin sensitivity in adults. Nutr Metab (Lond) 8(1), 48. doi: 10.1186/1743-7075-8-48.

Dawson, P.A., Lan, T., and Rao, A. (2009). Bile acid transporters. J Lipid Res 50(12), 2340-2357. doi: 10.1194/jlr.R900012-JLR200.

Deutz, N.E., Ten Have, G.A., Soeters, P.B., and Moughan, P.J. (1995). Increased intestinal amino-acid retention from the addition of carbohydrates to a meal. Clin Nutr 14(6), 354-364.

Ferezou, J., Riottot, M., Serougne, C., Cohen-Solal, C., Catala, I., Alquier, C., et al. (1997). Hypocholesterolemic action of beta-cyclodextrin and its effects on cholesterol metabolism in pigs fed a cholesterol-enriched diet. J Lipid Res 38(1), 86-100.

Fontana, L., Eagon, J.C., Trujillo, M.E., Scherer, P.E., and Klein, S. (2007). Visceral fat adipokine secretion is associated with systemic inflammation in obese humans. Diabetes 56(4), 1010-1013. doi: 10.2337/db061656.

Glicksman, C., Pournaras, D.J., Wright, M., Roberts, R., Mahon, D., Welbourn, R., et al. (2010). Postprandial plasma bile acid responses in normal weight and obese subjects. Ann Clin Biochem 47(Pt 5), 482-484. doi: 10.1258/acb.2010.010040.

Goodwin, B., Jones, S.A., Price, R.R., Watson, M.A., McKee, D.D., Moore, L.B., et al. (2000). A regulatory cascade of the nuclear receptors FXR, SHP-1, and LRH-1 represses bile acid biosynthesis. Mol Cell 6(3), 517-526.

Haeusler, R.A., Astiarraga, B., Camastra, S., Accili, D., and Ferrannini, E. (2013). Human insulin resistance is associated with increased plasma levels of 12alpha-hydroxylated bile acids. Diabetes 62(12), 4184-4191. doi: $10.2337 / \mathrm{db} 13-0639$.

Haeusler, R.A., Camastra, S., Nannipieri, M., Astiarraga, B., Castro-Perez, J., Xie, D., et al. (2016). Increased Bile Acid Synthesis and Impaired Bile Acid Transport in Human Obesity. J Clin Endocrinol Metab 101(5), $1935-1944$. doi: 10.1210/jc.2015-2583.

Haslewood, G.A. (1971). Bile salts of germ-free domestic fowl and pigs. Biochem J 123(1), 15-18.

Hata, S., Wang, P., Eftychiou, N., Ananthanarayanan, M., Batta, A., Salen, G., et al. (2003). Substrate specificities of rat oatp1 and ntcp: implications for hepatic organic anion uptake. Am J Physiol Gastrointest Liver Physiol 285(5), G829-839. doi: 10.1152/ajpgi.00352.2002.

Heuman, D.M. (1989). Quantitative estimation of the hydrophilic-hydrophobic balance of mixed bile salt solutions. $J$ Lipid Res 30(5), 719-730.

Hofmann, A.F. (1999). The continuing importance of bile acids in liver and intestinal disease. Arch Intern Med 159(22), 2647-2658.

Hofmann, A.F., and Hagey, L.R. (2008). Bile acids: chemistry, pathochemistry, biology, pathobiology, and therapeutics. Cell Mol Life Sci 65(16), 2461-2483. doi: 10.1007/s00018-008-7568-6.

Holt, J.A., Luo, G., Billin, A.N., Bisi, J., McNeill, Y.Y., Kozarsky, K.F., et al. (2003). Definition of a novel growth factordependent signal cascade for the suppression of bile acid biosynthesis. Genes Dev 17(13), 1581-1591. doi: 10.1101/gad.1083503.

Inagaki, T., Choi, M., Moschetta, A., Peng, L., Cummins, C.L., McDonald, J.G., et al. (2005). Fibroblast growth factor 15 functions as an enterohepatic signal to regulate bile acid homeostasis. Cell Metab 2(4), 217-225. doi: 10.1016/j.cmet.2005.09.001. 
Jain, A.K., Stoll, B., Burrin, D.G., Holst, J.J., and Moore, D.D. (2012). Enteral bile acid treatment improves parenteral nutrition-related liver disease and intestinal mucosal atrophy in neonatal pigs. Am J Physiol Gastrointest Liver Physiol 302(2), G218-224. doi: 10.1152/ajpgi.00280.2011.

Kawamata, Y., Fujii, R., Hosoya, M., Harada, M., Yoshida, H., Miwa, M., et al. (2003). A G protein-coupled receptor responsive to bile acids. J Biol Chem 278(11), 9435-9440. doi: 10.1074/jbc.M209706200.

Kim, I., Ahn, S.H., Inagaki, T., Choi, M., Ito, S., Guo, G.L., et al. (2007). Differential regulation of bile acid homeostasis by the farnesoid X receptor in liver and intestine. J Lipid Res 48(12), 2664-2672. doi: 10.1194/jlr.M700330JLR200.

Kong, B., Wang, L., Chiang, J.Y., Zhang, Y., Klaassen, C.D., and Guo, G.L. (2012). Mechanism of tissue-specific farnesoid $X$ receptor in suppressing the expression of genes in bile-acid synthesis in mice. Hepatology 56(3), 1034-1043. doi: 10.1002/hep.25740.

Kuipers, F., Bloks, V.W., and Groen, A.K. (2014). Beyond intestinal soap--bile acids in metabolic control. Nat Rev Endocrinol 10(8), 488-498. doi: 10.1038/nrendo.2014.60.

Kullak-Ublick, G.A., Stieger, B., Hagenbuch, B., and Meier, P.J. (2000). Hepatic transport of bile salts. Semin Liver Dis 20(3), 273-292. doi: 10.1055/s-2000-9426.

LaRusso, N.F., Hoffman, N.E., Korman, M.G., Hofmann, A.F., and Cowen, A.E. (1978). Determinants of fasting and postprandial serum bile acid levels in healthy man. Am J Dig Dis 23(5), 385-391.

Lefebvre, P., Cariou, B., Lien, F., Kuipers, F., and Staels, B. (2009). Role of bile acids and bile acid receptors in metabolic regulation. Physiol Rev 89(1), 147-191. doi: 10.1152/physrev.00010.2008.

Legrand-Defretin, V., Juste, C., Henry, R., and Corring, T. (1991). Ion-pair high-performance liquid chromatography of bile salt conjugates: application to pig bile. Lipids 26(8), 578-583.

Lu, T.T., Makishima, M., Repa, J.J., Schoonjans, K., Kerr, T.A., Auwerx, J., et al. (2000). Molecular basis for feedback regulation of bile acid synthesis by nuclear receptors. Mol Cell 6(3), 507-515.

Makishima, M., Okamoto, A.Y., Repa, J.J., Tu, H., Learned, R.M., Luk, A., et al. (1999). Identification of a nuclear receptor for bile acids. Science 284(5418), 1362-1365.

Maruyama, T., Miyamoto, Y., Nakamura, T., Tamai, Y., Okada, H., Sugiyama, E., et al. (2002). Identification of membrane-type receptor for bile acids (M-BAR). Biochem Biophys Res Commun 298(5), 714-719.

Miller, E.R., and Ullrey, D.E. (1987). The pig as a model for human nutrition. Annu Rev Nutr 7, 361-382. doi: 10.1146/annurev.nu.07.070187.002045.

Mok, H.Y., Von Bergmann, K., and Grundy, S.M. (1977). Regulation of pool size of bile acids in man. Gastroenterology 73(4 Pt 1), 684-690.

Parks, D.J., Blanchard, S.G., Bledsoe, R.K., Chandra, G., Consler, T.G., Kliewer, S.A., et al. (1999). Bile a cids: natural ligands for an orphan nuclear receptor. Science 284(5418), 1365-1368.

Prinz, P., Hofmann, T., Ahnis, A., Elbelt, U., Goebel-Stengel, M., Klapp, B.F., et al. (2015). Plasma bile acids show a positive correlation with body mass index and are negatively associated with cognitive restraint of eating in obese patients. Front Neurosci 9, 199. doi: 10.3389/fnins.2015.00199.

Roda, A., Minutello, A., Angellotti, M.A., and Fini, A. (1990). Bile acid structure-activity relationship: evaluation of bile acid lipophilicity using 1-octanol/water partition coefficient and reverse phase HPLC. J Lipid Res 31(8), 1433-1443.

Sato, H., Macchiarulo, A., Thomas, C., Gioiello, A., Une, M., Hofmann, A.F., et al. (2008). Novel potent and selective bile acid derivatives as TGR5 agonists: biological screening, structure-activity relationships, and molecular modeling studies. J Med Chem 51(6), 1831-1841. doi: 10.1021/jm7015864.

Schalm, S.W., LaRusso, N.F., Hofmann, A.F., Hoffman, N.E., van Berge-Henegouwen, G.P., and Korman, M.G. (1978). Diurnal serum levels of primary conjugated bile acids. Assessment by specific radioimmunoassays for conjugates of cholic and chenodeoxycholic acid. Gut 19(11), 1006-1014.

Schooneman, M.G., Ten Have, G.A., van Vlies, N., Houten, S.M., Deutz, N.E., and Soeters, M.R. (2015). Transorgan fluxes in a porcine model reveal a central role for liver in acylcarnitine metabolism. Am J Physiol Endocrinol Metab 309(3), E256-264. doi: 10.1152/ajpendo.00503.2014.

Steiner, C., Othman, A., Saely, C.H., Rein, P., Drexel, H., von Eckardstein, A., et al. (2011). Bile acid metabolites in serum: intraindividual variation and associations with coronary heart disease, metabolic syndrome and diabetes mellitus. PLoS One 6(11), e25006. doi: 10.1371/journal.pone.0025006.

Swindle, M.M., Makin, A., Herron, A.J., Clubb, F.J., Jr., and Frazier, K.S. (2012). Swine as models in biomedical research and toxicology testing. Vet Pathol 49(2), 344-356. doi: 10.1177/0300985811402846. 
Ten Have, G.A., Bost, M.C., Suyk-Wierts, J.C., van den Bogaard, A.E., and Deutz, N.E. (1996). Simultaneous measurement of metabolic flux in portally-drained viscera, liver, spleen, kidney and hindquarter in the conscious pig. Lab Anim 30(4), 347-358.

Twisk, J., Hoekman, M.F., Muller, L.M., lida, T., Tamaru, T., ljzerman, A., et al. (1995). Structural aspects of bile acids involved in the regulation of cholesterol 7 alpha-hydroxylase and sterol 27-hydroxylase. Eur J Biochem 228(3), 596-604.

van Nierop, F.S., Scheltema, M.J., Eggink, H.M., Pols, T.W., Sonne, D.P., Knop, F.K., et al. (2017). Clinical relevance of the bile acid receptor TGR5 in metabolism. Lancet Diabetes Endocrinol 5(3), 224-233. doi: 10.1016/S22138587(16)30155-3. 


\section{SUPPLEMENTAL MATERIALS}

\section{Detailed experimental overview bile acid analysis using LC/MS/MS}

\section{Chemicals:}

Cholic acid (CA), taurocholic acid (TCA), glycocholic acid (GCA), deoxycholic acid (DCA), taurodeoxycholic acid (TDCA), glycodeoxycholic acid (GDCA), chenodeoxycholic acid (CDCA), taurochenodeoxycholic acid (TCDCA), glycochenodeoxycholic acid (GCDCA), lithocholic acid (LCA), taurolithocholic acid (TLCA), ursodeoxycholic acid (UDCA) and glycoursodeoxycholic acid (GUDCA) were purchased from SigmaAldrich (St. Louis, MO). Glycolithocholic acid (GLCA) was purchased from Makaira Ltd (London, England). Tauroursodeoxycholic acid (TUDCA) was purchased from Merck Millipore (Billerica, MA). Hyodeoxycholic acid (HDCA), taurohyodeoxycholic acid (THDCA) and glycohyodeoxycholic acid (GHDCA) were purchased from Steraloids Inc (Newport, RI). D4-cholic acid (D4-CA), D4-chenodeoxycholic acid (D4-CDCA), D4glycochenodeoxycholic acid (D4-GCDCA) and D4-glycocholic acid (D4-GCA) were purchased from CDN Isotopes (Pointe-Claire, Quebec, Canada). D4-taurochenodeoxycholic acid (D4-TCDCA) and D4taurocholic acid (D4-TCA) were purchased from Medical Isotopes (Pelham, NH).

Instrumentation:

An LC-MS/MS system was used for the analysis of the plasma samples. The system consist of a SHIMADZU liquid chromatography (LC) system (SHIMADZU, Kyoto, Japan) coupled to a SCIEX API-3200 triple quadrupole mass spectrometer with electrospray ionization (ESI) source (SCIEX, Framingham, MA). The LC-MS/MS system is controlled by Analyst 1.6 software.

Liquid chromatographic and mass spectrometric conditions:

The mobile phase consisted of $20 \mathrm{mM}$ ammonium acetate, adjusted to $\mathrm{pH} 8.0$ with $25 \%$ ammonia (mobile phase A) and methanol (mobile phase B), at a total flow rate of $0.2 \mathrm{ml} / \mathrm{min}$. The gradient profile is shown in Supplementary Table 1. 
Table S1. Gradient profile.

\begin{tabular}{llll}
\hline Total time $(\min )$ & Flow rate $(\mathrm{mL} / \mathbf{m i n})$ & \% A & \% B \\
\hline 0 & 0.2 & 60 & 40 \\
\hline 24 & 0.2 & 20 & 80 \\
\hline 24.5 & 0.2 & 5 & 95 \\
\hline 26.5 & 0.2 & 5 & 95 \\
\hline 27 & 0.2 & 60 & 40 \\
\hline 30 & 0.2 & 60 & 40 \\
\hline
\end{tabular}

The injection volume of all samples was $10 \mu \mathrm{l}$.

Bile acids were separated using a XBridge TM Shield RP18 column (100 mm x $2.1 \mathrm{~mm}, 3.5 \mu \mathrm{m}$ ) equipped with a XBridge TM Shield RP18 guard column (10 mm x $2.1 \mathrm{~mm}, 3.5 \mu \mathrm{m}$ ) (Waters, Milford, MA). The mass spectrometer (MS) parameters, such as gas pressure, ion spray voltage, temperature, etc., were optimized by infusing each bile acid and the internal standards (IS) separate in a $50 \% \mathrm{MeOH}$ solution via a Harvard pump 11 standard infusion syringe pump (Harvard Apparatus, South Natick, MA). All bile acids were detected in negative mode with the mass spectrometer source settings shown in Supplementary Table 2 and 3.

Table S2. Mass spectrometer source settings (API-3200).

\begin{tabular}{ll}
\hline Detection mode & MRM, negative mode \\
\hline Resolution Q1 & Unit \\
\hline Resolution Q3 & Unit \\
\hline Curtain gas & 10 \\
\hline CAD gas & 10 \\
\hline Nebulizer gas (GS1) & 25 \\
\hline Heater gas (GS2) & 30 \\
\hline Temperature & $600{ }^{\circ} \mathrm{C}$ \\
\hline lon spray voltage (IS) & $-4500 \mathrm{~V}$ \\
\hline MR pause & $5 \mathrm{msec}$ \\
\hline
\end{tabular}

The multiple reaction monitoring (MRM) transitions for each bile acid and internal standard, as well as their optimum MS parameters such as collision energy (CE), declustering potential (DP), focusing 
potential (FP), cell exit potential (CXP) and cell entrance potential (CEP) are shown in Supplementary

Table 3.

Table S3. MRM settings API-3200.

\begin{tabular}{|c|c|c|c|c|c|c|c|c|}
\hline Component & $\begin{array}{l}\text { Q1 } \\
\text { mass }\end{array}$ & $\begin{array}{l}\text { Q3 } \\
\text { mass }\end{array}$ & DP & CEP & CE & CXP & EP & $\begin{array}{l}\text { Dwell } \\
\text { (msec) }\end{array}$ \\
\hline $\mathrm{CA}$ & 407.2 & 407.2 & -28 & -21 & -14 & -4 & -12 & 180 \\
\hline TCA & 514.2 & 79.9 & -39 & -25 & -121 & -1 & -4 & 180 \\
\hline GCA & 464.2 & 74.1 & -106 & -23 & -65 & -1 & -7 & 180 \\
\hline CDCA, DCA & 391.3 & 391.3 & -125 & -19 & -14 & -5 & -8 & 180 \\
\hline TCDCA, TDCA & 498.4 & 79.9 & -150 & -23 & -113 & -2 & -7 & 180 \\
\hline GCDCA, GDCA & 448.4 & 74.0 & -102 & -20 & -62 & -1 & -7 & 180 \\
\hline UDCA, HDCA & 391.3 & 391.3 & -130 & -26 & -13 & -4 & -4 & 180 \\
\hline TUDCA, THDCA & 498.4 & 79.9 & -23 & -21 & -83 & -1 & -5 & 180 \\
\hline GUDCA, GHDCA & 448.4 & 74.0 & -118 & -20 & -63 & -1 & -10 & 180 \\
\hline LCA & 375.1 & 375.1 & -123 & -18 & -13 & -6 & -7 & 180 \\
\hline TLCA & 482.2 & 79.8 & -60 & -22 & -109 & -1 & -7 & 180 \\
\hline GLCA & 432.1 & 74.0 & -100 & -22 & -58 & -2 & -4 & 180 \\
\hline D4-CA & 411.2 & 411.2 & -28 & -21 & -14 & -4 & -12 & 180 \\
\hline D4-TCA & 518.2 & 79.9 & -39 & -25 & -121 & -1 & -4 & 180 \\
\hline D4-GCA & 468.2 & 74.1 & -106 & -23 & -65 & -1 & -7 & 180 \\
\hline D4-CDCA & 395.3 & 395.3 & -134 & -19 & -13 & -5 & -10 & 180 \\
\hline D4-TCDCA & 502.4 & 79.9 & -146 & -23 & -111 & -1 & -7 & 180 \\
\hline D4-GCDCA & 452.4 & 74.0 & -102 & -20 & -62 & -1 & -7 & 180 \\
\hline
\end{tabular}

